\title{
VARIATIONAL CALCULATIONS IN QUANTUM FIELD THEORY
}


This publication is the second volume in a series devoted to the Proceedings of the International Workshops on the Physics of Small Systems. Editor-in-Chief: E.R.Hilf (University of Oldenburg, FRG) 
Proceedings of the International Workshop on

\section{VARIATIONAL CALCULATIONS IN QUANTUM FIELD THEORY}

Wangerooge, West Germany 1-4 September 1987 


\section{Published by}

World Scientific Publishing Co. Pte. Ltd.

P.O. Box 128, Farrer Road, Singapore 9128

U. S. A. office: World Scientific Publishing Co., Inc.

687 Hartwell Street, Teaneck NJ 07666, USA

\section{VARIATIONAL CALCULATIONS IN QUANTUM FIELD THEORY \\ Copyright $\odot 1988$ by World Scientific Publishing Co Pte Ltd. \\ All rights reserved. This book, or parts thereof, may not be reproduced in any form or by any means, electronic or mechanical, including photo- copying, recording or any information storage and retrieval system now known or to be invented, without written permission from the Publisher.}

ISBN 9971-50-500-2

Printed in Singapore by JBW Printers \& Binders Pte. Ltd. 
This Proceedings is dedicated to the memory of R.P.Feynman who devoted much of his research to the variational principle in field theory and who shared his intuition with the participants of this workshop.

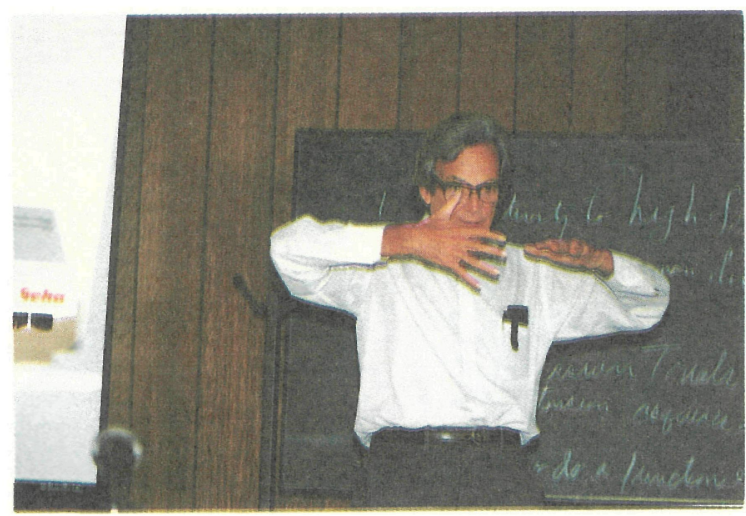


This page is intentionally left blank 


\section{Preface}

For a long time now there has been considerable interest in the possibility of obtaining accurate qualitative and quantitative information on the structure of quantum field theories using non-perturbative continuum and lattice variational techniques. This workshop brought together for the first time the leading researchers in this field in order to review its current state-of-art as well as to identify profitable directions for future research.

The themes of the meeting were: the Schrödinger formulation of QFT, the incorporation of gauge-fixing and renormalization, finite-mode and Gaussian approximations, the systematic improvement of trial states, investigations of vacuum instabilities and applications to scalar, gauge and supersymmetric theories.

The organizers would like to thank the participants for their encouragement before and during the meeting, and for providing a lively and informal workshop atmosphere.

It is a pleasure to thank our workshop secretary, Juliane Hilf, for her efficient handling of the day-to-day conference business. Furthermore, we are grateful to Uwe Ritschel and Michael Wendel for technical assistance, to Paulina Mendel and Theresia Meyer for retyping some of the contributions in $\mathrm{BT}_{\mathrm{E}} \mathrm{X}$, and to the staff of the Computer Center at Oldenburg University for their assistance in using $\mathrm{I}_{\mathrm{E}} \mathrm{T}_{\mathrm{E}} \mathrm{X}$ and the printing facilities.

We gratefully acknowledge the technical support we received from the community of Wangerooge island, especially through Mr. G.Till, Mr. W.Janssen, Mrs. A.Haupt, and Mr. R.-E.Jakobs.

The workshop was generously funded by the Stiftung Volkswagenwerk with some additional support taken from a grant of the Bundesministerium für Forschung und Technologie.

Oldenburg/Winchester, May 1988

Eberhard Hilf

Editor-in-Chief
Lutz Polley

David Pottinger 
This page is intentionally left blank 


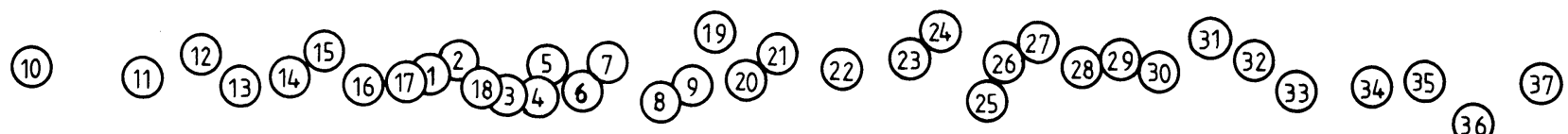

$\begin{array}{llllllll}1 & \text { G.Preparata } & 10 & \text { A.Krasnitz } & 20 & \text { W.Wollny } & 30 & \text { U.Schermuly } \\ 2 & \text { R.Tarrach } & 11 & \text { D.Schütte } & 21 & \text { W.A.Bardeen } & 31 & \text { U.Ritschel } \\ 3 & \text { L.Polley } & 12 & \text { J.Greensite } & 22 & \text { D.Horn } & 32 & \text { K.Hein } \\ 4 & \text { E.Klepfish } & 13 & \text { R.E.Cutkosky } & 23 & \text { R.P.Feynman } & 33 & \text { A.Patkós } \\ 5 & \text { A.Kovner } & 14 & \text { B.Rosenstein } & 24 & \text { W.Dykshoorn } & 34 & \text { C.Cronström } \\ 6 & \text { D.Pottinger } & 15 & \text { A.Duncan } & 25 & \text { K.Yamazaki } & 35 & \text { P.Besting } \\ 7 & \text { J.Wudka } & 16 & \text { J.E.Mandula } & 26 & \text { J.Hilf } & 36 & \text { Mrs. Oleszczuk } \\ 8 & \text { F.Grassi } & 17 & \text { O.Eyal } & 27 & \text { J.W.Darewych } & 37 & \text { M.Oleszczuk } \\ 9 & \text { Mrs. Bardeen } & 18 & \text { T.Kanki } & 28 & \text { M.Wendel } & & \\ & & 19 & \text { E.R.Hilf } & 29 & \text { M.Horbatsch } & & \end{array}$


This page is intentionally left blank 


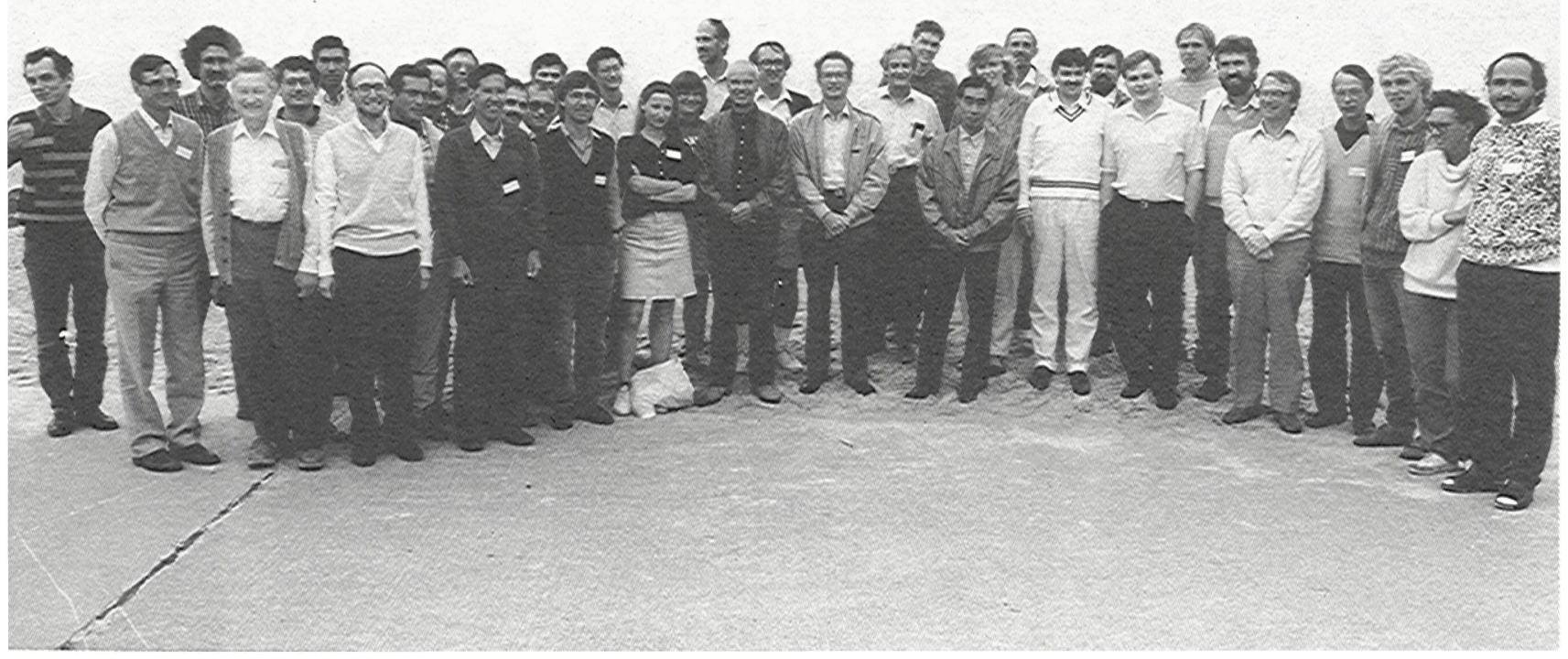


This page is intentionally left blank 


\section{Participants}

William A. BARDEEN, Theoretical Physics Department, Fermi National Accelerator Laboratory, P.O.Box 500, Batavia, IL 60510, U.S.A.

Peter BESTING, Institut für Theoretische Kernphysik, Universität Bonn, Nußallee 14-16, 5300 Bonn, West Germany

Roberto CASALBUONI, Dipartimento di Fisica dell'Universitá di Lecce, 73100 Lecce, Italy; and INFN, Sezione di Bari, 70126 Bari, Italy

Cristofer CRONSTRÖM, Dept. of Theoretical Physics, University of Helsinki, Siltavuorenpenger 20C, 00170 Helsinki, Finland

Richard E. CUTKOSKY, Physics Dept., Carnegie-Mellon University, Schenley Park, Pittsburgh, PA 15213, U.S.A.

Jurij W. DAREWYCH, Dept. of Physics, York University, Toronto, Ontario, Canada M3J 1P3

Anthony DUNCAN, Dept. of Physics and Astronomy, University of Pittsburgh, Pittsburgh, PA 15260, U.S.A.

William P. DYKSHOORN, Physics Dept., York University, 4700 Keele Street, North York, Ontario, Canada M3J 1P3

Ofer EYAL, Physics Dept., Technion, Haifa 32000, Israel

Richard P. FEYNMAN, California Institute of Technology, Pasadena, CA 91125, U.S.A.

Frédérique GRASSI, M.S.209, Fermilab, P.O.B. 500, Batavia, IL 60510, U.S.A.

Jeffrey P. GREENSITE, Dept. of Physics and Astronomy, San Francisco State University, 1600 Holloway Ave., San Francisco, CA 94132, U.S.A.

Klaus HEIN, Institut für Theoretische Physik, Universität Heidelberg, Philosophenweg 16, 6900 Heidelberg, West Germany

Eberhard HILF, Fachbereich Physik, Universität Oldenburg, Postfach 2503, 2900 Oldenburg, West Germany

Marko HORBATSCH, Dept. of Physics, York University, Toronto M3J 1P3, Canada

David HORN, School of Physics and Astronomy, Tel Aviv University, Tel Aviv 69978, Israel 
Takeshi KANKI, The Second Department, Faculty of Science and Technology, Kinki University, Higashi-Osaka, Osaka 577, Japan

Elyakum KLEPFISH, School of Physics and Astronomy, Tel Aviv University, Ramat Aviv 69978, Israel

Roman KONIUK, Dept. of Physics, York University, Toronto, Ontario, Canada M3J 1P3

Alexander KOVNER, High Energy Physics Dept., School of Physics and Astronomy, Tel Aviv University, Ramat Aviv 69978, Israel

Alexander KRASNITZ, School of Physics and Astronomy, Tel Aviv University, Ramat Aviv 69978, Israel

Jeffrey E. MANDULA, Physics Research Branch, Division of High Energy Physics, Department of Energy, Washington, DC 20545, U.S.A.

Michael OLESZCZUK, Institut für Physik I - Theoretische Physik, Universität Regensburg, Universitätsstraße 31, 8400 Regensburg, West Germany

András PATKÓS, Dept. of Atomic Physics, Eötvös University, 1088 Budapest, Puskin u. 5-7, Hungary

Lutz POLLEY, Fachbereich Physik, Universität Oldenburg, Postfach 2503, 2900 Oldenburg, West Germany

David POTTINGER, IBM, UK Scientific Centre, Athelstan House, St.Clement Street, Winchester, Hampshire SO23 9DR, U.K.

Giuliano PREPARATA, Dipartimento di Fisica - Universitá di Milano, INFN - Sezione di Milano, 20133 Milano, Italy

Uwe RITSCHEL, Fachbereich Physik, Universität Oldenburg, Postfach 2503, 2900 Oldenburg, West Germany

Baruch ROSENSTEIN, Center for Particle Theory, Dept. of Physics, University of Texas, Austin TX 78712, U.S.A.

Ulrich SCHERMULY, Fachbereich Physik, Technische Hochschule Darmstadt, 6100 Darmstadt, West Germany

Dieter SCHÜTTE, Institut für Theoretische Kernphysik, Universität Bonn, Nußallee 14-16, 5300 Bonn, West Germany

Rolf TARRACH, Dep. Estructura i Constituents de la Matèria, Univ. de Barcelona, Diagonal 647, 08028 Barcelona, Spain 
Michael WENDEL, Fachbereich Physik, Universität Oldenburg, Postfach 2503, 2900 Oldenburg, West Germany

Wolfgang WOLLNY, Fachbereich Mathematik,

Technische Hochschule Darmstadt, 6100 Darmstadt, West Germany

José WUDKA, Randall Laboratory of Physics, The University of Michigan, Ann Arbor, MI 48109-1120, U.S.A.

Kazuo YAMAZAKI, Dept. of Physics, College of Liberal Arts and Sciences, Kyoto University, Kyoto 606, Japan 
This page is intentionally left blank 


\section{Contents}

A Variational Approach to QCD Bound States 1 by J.Greensite

Vacuum and Excited States

in Truncated-Mode Coulomb-Gauge Yang-Mills Theory

by R.E.Cutkosky and K.C.Wang

Difficulties in Applying the Variational Principle

to Quantum Field Theories

by R.P.Feynman

Schrödinger Approach to Ground State Wavefunctionals by W.A.Bardeen

Computer Measurement of the Yang-Mills Vacuum (and String) Wavefunctionals by J.Greensite

The Magnetic Instability of the Perturbative Yang-Mills Vacuum 63 by G.Preparata

A Step Beyond the Gaussian Approximation in Yang-Mills Theory 75 by L.Polley and U.Ritschel

Dynamical Symmetry Breaking in QCD and Quark Masses

by R.Casalbuoni

Zero Temperature Quark Matter Equation of State by F.Grassi

$\lambda \Phi_{3+1}^{4}, \lambda>0$, with Spontaneous Symmetry Breaking by R.Tarrach

Gaussian Approximations and Renormalization of Effective Potentials in $1+1$ vs Higher Dimensions by J.Wudka

Covariant Gaussian Approximation: Formalism by A.Kovner and B.Rosenstein

Covariant Gaussian Approximation: Applications 
Variational Methods in Large N Supersymmetric Models by O.Eyal

Variational Calculations of Particle Masses in Quantum Field Theory: Beyond the Simplest Gaussian Ansatz 172 by J.W.Darewych, M.Horbatsch and R.Koniuk

Bound State Wavefunctions in Strongly Coupled QED 188 by W.Dykshoorn, R.Koniuk and J.W.Darewych

Feynman's Polaron Theory:

Translation into Hamiltonian Language by K.Yamazaki

Asymptotics of Classical Yang-Mills Fields

by C.Cronström

Variational Calculation of Instanton-Based Yang-Mills Vacuum at Finite Temperature 215 by T.Kanki

The Structure of Gauge Field Theories within

Different Regularization Schemes and Aspects of Confinement by P.Besting, J.Neubauer and D.Schütte

The Deconfined Phase of QCD by J.E.Mandula and M.Ogilvie

Spectral Estimates for Hamiltonian Lattice Theory by A.Duncan

Testing Variational Ground States Against Conformal Invariance 254 by A.Patkós

The t-Expansion in Lattice QCD 266

by D.Horn

Ground State

of Lattice SU(2) QCD with Finite Chemical Potential by E.G.Klepfish and A.Krasnitz 


\section{VARIATIONAL CALCULATIONS IN QUANTUM FIELD THEORY}

